\title{
Analysis on Fatigue Elimination of Football Training
}

\author{
Feng Du \\ Xi 'an Peihua college, Xi’an, Shaanxi, 710125
}

Keywords: Fatigue Elimination, Football Training, Sports Technology

\begin{abstract}
Exercise fatigue refers to the physical process of the body can not continue its function at a particular level or can not maintain the intended exercise intensity and physical incapacity. Moderate fatigue, impose a scientific and reasonable means of recovery, can promote the body function and improve the level of sports technology. And excessive exercise fatigue and inappropriate means of recovery, not only is not conducive to the improvement of athletic performance, and may form a sports injury, which does not meet the purpose of sports training. Therefore, this article for the football exercise training in the emergence of sports fatigue research, understanding and understanding of the mechanism of fatigue, master reasonable and effective means of recovery, so as to eliminate the impact of fatigue, to help improve the training level.
\end{abstract}

\section{Introduction}

The main trend of modern football is the individual, local and overall technology, tactical system more and more sophisticated and highly targeted, more and more intense confrontation. How to use the technical tactical tactics in a fast, reasonable and skillful way in the case of high confrontation competition, often determines the control of the game or even the outcome. Therefore, according to the concept of modern football, imitation of the real situation of the game organization training to become the trend of football training, the quality of the training for the players and the conditions of the competition under the conditions of the requirements are getting higher and higher, which is the football player's physical training A higher demand. In the fierce competition and high-intensity training, the athletes in the physical and psychological aspects of the signs of fatigue, if not timely recovery will lead to athletes physical function, psychological and other competitive ability of the decline, restricting the level of athletes training The improvement. To this end, from the solution to the existing problems of the movement of the project, find the mechanism of fatigue and prevention measures to improve the level of football training, improve athletes competitive ability to guide.

\section{The Importance of Football Characteristics and Physical Fitness}

Statistics show that the 1998 World Cup players in a game running distance of $9000 \sim 14000 \mathrm{~m}$, an average of $10800 \mathrm{~m}$. With the establishment of modern football full attack keep the type of play, the competition requires the athletes to carry out cross transposition, complement each other, random control, repeated sprint run, in the fierce confrontation in the fast completion of skills, tactical action hundreds of times, the game Not only the strength of large, and the density between each game is also large, the level of athletes training requirements are getting higher and higher, the athlete's physical fitness level in the game is very important. Within 90 min or even 120 min, the athletes if there is no good physical training level, it will lead to physical, mental, emotional, emotional aspects of the decline in physical function, the wrong action increased, it is difficult to play a high degree of technical and tactical level The Therefore, athletes must have a very good physical fitness to meet the needs of the game. Physical skills is the basis of skills, skills are physical expression, only in the physical and skills and good psychological quality assurance can be completed under a variety of tactical arrangements. Therefore, good physical fitness and plenty of physical strength is the athlete in the game has always been to maintain high-speed athletic ability of the premise and guarantee. 
Nearly several World Cup in the game at the end of the time the most into the ball, which shows that after a long period of intense scraping, the player's physical strength decreased significantly, attention can not be highly concentrated, to the offensive side to create an opportunity. According to statistics, the current world professional football players running the average distance of each game in the $9000 \sim 14000 \mathrm{~m}$ or so, coupled with the frequent variety of events, making athletes physical consumption is huge. If the recovery between each game and training is not complete, easy to produce excessive fatigue eventually lead to competitive decline and injury events occur frequently. Therefore, how to eliminate fatigue as soon as possible to restore physical fitness, has become another focus of today's football training.

\section{Football Player Fatigue Mechanism}

Soccer is a combination of intermittent, high-intensity repeated sprint and a long time for the characteristics of intense scraping to oxygen for the original energy supply and phosphate-based mixed sports. Competition in the high-intensity work requires the original supply of phosphoric acid, after the recovery of CP and lactic acid to eliminate the speed, but also depends on the level of aerobic metabolism of muscle. Therefore, in the movement, when the energy consumption to a certain extent and can not add recovery, the body's function will be disorder, exercise capacity also will be reduced; at the same time in the course of the movement will continue to produce metabolites, such as a lot of sweat exclusion, salt Of the loss and the accumulation of a large number of lactic acid, making muscle activity disorders and fatigue. In a football training course, with the acceleration of the conversion, requiring practitioners in the field to a large range of high-speed running to participate in the attack and defense, in the control of the ball at the same time, often urgent emergency, anxious to get rid of defense, and The body of the confrontation is inevitable, so that the body in a long time aerobic and short hours of oxygen-free working environment. In the short-term anaerobic muscle strong contraction, the body is the consumption of phosphate material, in the body about 30mins anaerobic endurance work, consumption of glycogen. In the long hours of aerobic work, the body is the consumption of fat for energy, but in the process of lipolysis to have the participation of sugar in the sugar reserve is not sufficient, the lipolysis process can not be carried out. Sugar is an important energy during exercise, in the blood and other extracellular fluid glucose storage is about $20 \mathrm{~g}$. Heavy load movement can make the blood sugar content of the rapid decline. Therefore, the liver must continue to break down glycogen into glucose into the blood, to prevent hypoglycemia and lead to fatigue. When the glycogen is consumed in large quantities, the speed of movement is reduced, which is the main reason for the long-term muscle movement to produce fatigue.

A heavy load training class, practitioners to carry out a lot of technical, tactical and physical quality of practice, in order to adapt to the rapid attack and defense conversion, the field running fast, large range. A single technique in the overall tactical use of many times, practitioners have to complete the special speed, strength, endurance and other physical fitness exercises. In order to complete these exercise loads, the consumption of energy in the body will produce a lot of waste such as lactic acid, $\mathrm{H}+$, free radicals and other substances, more than the transport capacity of the blood and the formation of waste accumulation, these metabolites will bring some negative effects: ) Inhibit the decomposition of glycogen, inhibit adipose tissue lipolysis, increase muscle moisture content and reduce lactic acid from the muscle out.

(2) The acidic environment reduces the sensitivity of tissues and organs to changes in nerve impulses and local microenvironment, reduces troponin and $\mathrm{Ca} 2+$ binding, increases $\mathrm{Ca} 2+$ requirements, reduces ATPase activity, increases protein binding to $\mathrm{Ca} 2+$, increases $\mathrm{K}+$ Extracellular fluid concentration, reduce muscle tissue maximum tension and sustained ability to extend the muscle refractory period, reduce the nerve tissue excitability, resulting in fatigue.

The Pavlov school believes that exercise-induced fatigue is due to the protective inhibition of the cerebral cortex, a large number of impulses transmitted to the cerebral cortex corresponding to the nerve cells, so that prolonged excitement led to increased consumption, when consumed to a certain extent The inhibition, in order to prevent excessive body failure. As the football needs to respond in 
a very short period of time, and need to repeat the high intensity of exercise, often causing excessive cortical cortex and excessive inhibition, if the nervous system engaged in excessive exercise, exercise time is too long, It is easy to destroy the original power stereotypes, easily lead to cortical function disorders, resulting in dysfunction of the various organs of the system.

With the increase in the intensity of practice, attack, anti-rhythm of the accelerated, practitioners to complete a variety of technical movements and tactical cooperation at the same time, sometimes there is no concentration of attention to the changes in the field is relatively slow, mistakes gradually increased, Judgment is not timely and inaccurate, it is due to the late stages of the football training course, accompanied by the consumption of energy substances in the practitioner's body, will produce a lot of lactic acid in the body, lactic acid and ATP and glycogen in the body of the product, And the accumulation of $\mathrm{H}+$ in the body will change the body's $\mathrm{pH}$. The stability of the environment within the body by the control of the nervous system, by hormones and other regulatory role through the body to maintain. Muscle activity by the nervous system dominance, whether it is random action or reflexive action, are the cerebral cortex nerve center of the nerve activity. Neurons are sensitive to the $\mathrm{pH}$ of the body fluids, lack of glycogen and oxygen, and because of the reduced blood flow to the cerebral cortex and the stimulation of the acidic metabolites in the blood, the function of the brain is reduced and the consciousness and consciousness are inhibited. Technical action when the slow response, action specifications are not in place and so on.

\section{Means of Fatigue Elimination}

In soccer training to take some measures to reduce the fatigue or reduce the degree of fatigue. Such as the understanding of the physical condition of athletes, the scientific development of training content and plans; the use of scientific and advanced training methods and methods of reasonable arrangements for exercise and exercise intensity; appropriate arrangements for the game; in the special practice-based principles of non-special practice; The rest of the gap, interspersed with some minor activities and so on.

The principle of resumption of training is to enable players to recover quickly and physically and physically, so that they can be put into more effective normal training and competitions as soon as possible. Players in the intense training and competition, there is no time and energy to communicate and communicate with each other, resume training classes can give players the opportunity to provide such a chance, it can play a positive role in the morale of the team, regular rest To avoid overloading the player. If the player can maintain physical fitness for a long time, to achieve the possibility of individual training goals is even greater, the greater the load, the more need to pay attention to timely recovery. Coaches should have a sensitive sense of consciousness, and must be flexible to use in order to ensure the players in the psychological and physical recovery on the rapid. Restoration of football training include: recovery after the game, the resumption of the race, after the resumption of training, after a week of training and so on.

In the football, both the need for anaerobic metabolism for energy and also requires the level of aerobic metabolism, of which the aerobic oxidation of glycogen and ATP-CP for energy-based. According to the Nanjing University of Science and Technology Sports Department Xia Qiang and others "experimental study - football players to add sugar or creatine on the impact of blood lactic acid after exercise" article, we can simply add oligosaccharides can significantly reduce the level of blood lactic acid after the game, At the same time to strengthen the athletes phosphate reserves, improve the ability of glycolysis capacity, so as to improve athletic competitions. Therefore, timely sugar is very important.

It is best to achieve $60 \%$ to $70 \%$ or $10 \mathrm{~g}$ kg body weight of total calorie intake during the first few days of competition or training, and 1 to $4 \mathrm{~g} \mathrm{~kg}$ Weight (note that $1 \mathrm{~h}$ before the game should be added liquid sugar). In the game every $30 \sim 60$ min should be added sugar-containing drinks or easy to absorb sugar-containing items, sugar should generally be $60 \mathrm{~g} \mathrm{~h}$ or $1 \mathrm{~g}$ min. The study found that oligosaccharide beverages are ideal nutrients in football, oligosaccharides can make the body in a long period of exercise to maintain a high level of blood sugar, does not cause blood contraction or 
serum insulin concentration increased. Electrolysis in the beverage is conducive to stable exercise in the serum magnesium level, does not cause increased serum potassium concentration is also conducive to reduce the level of blood lactate in exercise, therefore, it can increase the internal force, prolong the exercise time. The end of the game sugar supplement the sooner the better, the ideal time should be immediately after the game $2 \mathrm{~h}$ and every $1 \sim 2 \mathrm{~h}$ continuous supplement, the amount should be $0.75 \sim 1.00 \mathrm{~kg}$ is appropriate, because within 6h after the game Glycogen synthase content is high, glycogen recovery effect is good, $24 \mathrm{~h}$ after the game the total amount of sugar should be $9 \sim 16 \mathrm{~g}$ kg body weight. Glucose absorption of the fastest, most conducive to the synthesis of muscle glycogen, oligosaccharides due to osmotic pressure is only glucose 14 , the sweetness of the small should be quickly absorbed.

\section{Conclusion}

With the growing popularity of football, the world's football has been further developed, the level of football are generally get different degrees of improvement. However, in the development and improvement process also appeared some problems. Some soccer players in the training or competition to participate in the competition, often training or competition has just started to half began to feel powerless, there is physical fatigue, affecting the development of personal technology and the team's overall tactical implementation, so that Athletic level decline and affect the athletic performance, improve the level of exercise. Therefore, it is an important task to understand the mechanism of soccer athletes' fatigue fatigue, to grasp the reasonable, effective and scientific way to eliminate the fatigue of movement, to eliminate fatigue in a timely and complete way.

\section{References}

[1] Zhao Yufeng. Soccer athletes physical training content and methods of research [J]. Think tank era. 2017 (05)

[2] Deng Qian. Study on the Influence of Vibratory Stretching on Flexibility and Intermittent High Intensity Sprint of Soccer Players [J]. Journal of Shandong Institute of Physical Education, 2017 (04)

[3] Qiu Bobo. Effects of foam shaft relaxation on athletic performance of soccer players [J]. Movement. 2016 (19)

[4] Yang Yiren, Zhang Xinrui, Tao Xiaobo. Soccer player brand equity analysis [J]. Sports Culture Guide. 2016 (04)

[5] Liang Zhihui. Soccer player's emotions in the role of the game [J]. Tourism overview (the second half). 2014 (01)

[6] Zhang Yan. Soccer player control in the game tactical influence [J]. Contemporary sports technology. 2013 (24) 